\title{
La clasificación documental y sus aportes a la gestión documental: transparencia y acceso a la información en Uruguay
}

\author{
Stella Infante Rosso ${ }^{1}$
}

\section{Resumen}

La clasificación documental es un pilar esencial en la gestión archivística. Permite identificar, representar y reflejar al productor en su fondo documental a través de una estructura lógica y jerárquica como lo es el cuadro de clasificación documental, que proporciona y facilita el acceso a la información pública.

El presente trabajo pretende evaluar la situación actual de la clasificación documental a nivel del Sistema Nacional de Archivos en Uruguay, a diez años de promulgada la Ley 18220, que creó dicho sistema. A través del relevamiento efectuado se puede tener una noción del estado de situación de los archivos públicos en Uruguay, identificar los logros y dificultades ante los que se enfrentan los sujetos obligados por la ley para alcanzar una gestión documental profesional que garantice el acceso a la información pública, la transparencia y la rendición de cuentas del Estado.

Palabras clave: Clasificación; Cuadro de clasificación; Acceso a la información; Transparencia; Sistema Nacional de Archivos

Document classification and its contributions to document management: transparency and access to information in Uruguay

\begin{abstract}
Document classification is an essential pillar in archival management. It allows us to identify, represent and reflect the producer in his fonds through a logical and hierarchical structure such as the Document Classification Scheme, allowing and facilitating access to public information.

This work intends to assess the current situation of document classification at the National Archives System level in Uruguay, ten years after Law 18220 , which created said system, was enacted. Through the survey carried out, it is possible to have an idea of the status of the public archives in Uruguay, identifying the achievements and difficulties faced by the subjects bound by law in order to achieve a professional document management,

\footnotetext{
Profesora adjunta del Departamento Tratamiento y Transferencia de la Información. Instituto de Información, Facultad de Información y Comunicación, Universidad de la República (Udelar) (Uruguay). Correo electrónico: stella.infante@fic.edu.uy
} 
guaranteeing access to public information, transparency and accountability of the State.

Keywords: Classification; Classification scheme; Access to Information, Transparency; National Archives System.

\section{Introducción ${ }^{2}$}

Son varias las razones para trabajar específicamente sobre este tema. Desde que en Uruguay existe un marco normativo favorable a la gestión archivística, a diez años de la promulgación de la Ley 18220 (2007) que crea el Sistema Nacional de Archivos (de aquí en más, SNA), era necesario obtener datos concretos sobre el estado de situación de la clasificación documental en el marco del SNA, ya que al momento no existía ningún estudio o estadística que brindara cifras concretas sobre ello, y a su vez, resaltar la importancia de la clasificación documental como pilar en el desarrollo e implementación de una eficiente y profesional gestión documental.

El literal b del artículo 6 del Decreto 355/012 (2012), que reglamenta la Ley 18220 (2007), deja clara la responsabilidad de los sujetos obligados por la ley (instituciones públicas a nivel nacional) y establece que es su deber «realizar una organización científica de los documentos en soporte tradicional o electrónica, mediante la organización de los archivos de Gestión, Central e Histórico». Es sabido que esa organización empieza por una identificación y clasificación del fondo documental.

Es claro que los nuevos contextos normativos a nivel nacional e internacional implican una inclusión de la informática en la gestión administrativa y documental, además de garantizar las respuestas inmediatas a las solicitudes de acceso a la información pública que, en Uruguay, están reguladas por la Ley 18381 (2008). Estas necesitan de la presencia de la archivística para que aporte desde sus procesos y herramientas al logro de archivos organizados y accesibles que garanticen a los ciudadanos el ejercicio pleno de la democracia, la transparencia y la rendición de cuentas por parte del Estado.

Como lo han esbozado en el 2014 las archivistas españolas del Gobierno de Asturias María del Carmen Fernández Vega, Alicia Hernández Martín y María Belén de los Toyos de Castro,

desde el punto de vista de la práctica archivística, la normalización del proceso de clasificación se ha abordado, en la mayoría de las

2 La presente investigación es producto del trabajo final correspondiente al Máster en Gestión Documental, Transparencia y Acceso a la Información que imparte la Escuela Superior de Archivísitica y Gestión de Documentos (Esaged) de la Universidad Autónoma de Barcelona, España. Directora de tesis: Dra. Remei Perpinyà Morera. 
ocasiones, desde una perspectiva teórica, enfocada en la construcción de modelos de cuadros de clasificación basados en estructuras organizativas cambiantes, y no focalizada en la identificación y análisis de las funciones atribuidas a cada organización. A pesar de estos esfuerzos normalizadores, la realidad nos demuestra que no existe un modelo único de sistema de clasificación y que las actividades de clasificación siguen ejecutándose sin una metodología concreta (2014, p. 194).

En esta ocasión, se busca obtener un conocimiento fidedigno de la situación actual en materia de transparencia y acceso a la información en Uruguay clasificación documental, así como generar un análisis detallado de dicha situación, que permita establecer algunas pautas, acompasadas a la realidad nacional, que puedan ser una guía para facilitar la elaboración de cuadros de clasificación documentales en el ámbito del SNA uruguayo.

\section{Metodología}

La presente investigación fue realizada a través de un análisis cuantitativo. Para obtener los datos necesarios se diseñó una encuesta que pretende brindar datos básicos sobre cuál es el tratamiento que se les da a los fondos documentales en el marco del SNA, con especial foco en el desarrollo de sus cuadros de clasificación, así como en el conocimiento de sus elementos y la metodología utilizada en su elaboración.

En esta ocasión, se escogió como muestra representativa a los sujetos que cumplieran con las condiciones de ser profesionales archivólogos o estudiantes avanzados de dicha carrera y que ejercieran en instituciones obligadas por la ley del SNA, en el entendido de que serían ellos quienes podrían responder con propiedad ante la aplicación y el desarrollo de herramientas y procedimientos propios de la archivística. Se generó un así muestreo intencional, ya que los encuestados fueron seleccionados directamente.

De un total de 264 sujetos obligados de la ley, fueron entrevistados 40 profesionales $^{3}$ que se desempeñan en instituciones de la administración central, organismos descentralizados del Estado, entes autónomos y gobiernos departamentales.

La encuesta fue aplicada entre julio y setiembre de 2017, en forma telefónica o presencial, de acuerdo con las disponibilidades horarias de los encuestados, y en los casos en los que no fue posible contactar al profesional mediante las vías de comunicación mencionadas, se envió por correo electrónico el formulario. Así, el $85 \%$ de las encuestas fue presencial o mediante entrevista telefónica, en el entendido de que en la conversación directa con el encuestado se alcanzan mayores datos y de mejor calidad, ya

3 Profesionales que poseen el título de Archivólogo/a o de Licenciado/a en Archivología, aunque en algunos casos no tengan reconocida su titulación ante la autoridad institucional. 
que se direcciona la entrevista, atendiendo algunas dudas surgidas a la hora de contestar el cuestionario.

La muestra relevada constituye un $15 \%$ del total de la población antes definida. El cuadro 1 representa la distribución de los encuestados de acuerdo con la pertenencia a los diferentes órdenes del Estado, según su naturaleza jurídica. Vale resaltar que la mayoría de los encuestados están en Montevideo, la capital del país, donde está centralizada la mayor parte de las funciones del Estado. Así mismo, se pudo contactar a tres colegas del interior para integrar esta muestra, lo cual denota la falta de profesionales archivólogos en el interior del país, perteneciendo estos a archivos de gobiernos departamentales.

\begin{tabular}{l|c|}
\cline { 2 - 2 } Poder Legislativo & 1 \\
\hline Poder Judicial & 1 \\
\hline Poder Ejecutivo & 23 \\
\hline Entes Autónomos & 7 \\
\hline Servicios Descentralizados & 3 \\
\hline Gobiernos Departamentales & 4 \\
\hline Personas públicas no estatales & 1 \\
\hline
\end{tabular}

\section{Cuadro 1.}

Distribución de la muestra por órdenes del Estado. Elaboración propia

El cuestionario utilizado en la entrevista estaba conformado por 33 preguntas divididas en 4 bloques temáticos:

1. Datos de contacto del encuestado. En esta sección se logró tener un conocimiento del perfil del encuestado y de la muestra en general.

2. Sobre el archivo. En esta sección se buscó obtener datos básicos sobre el archivo institucional, su funcionamiento y el personal afectado a él, y de esta manera conocer si se cumplen aspectos como la profesionalización y el tratamiento de estos archivos institucionales de acuerdo con las disposiciones de la Ley 18220 (2007), especialmente con lo dispuesto en los artículos 1 y 7. En dichos artículos queda establecido, el deber y la responsabilidad del Estado sobre que «la conservación y organización del Patrimonio Documental de la Nación [...] deberá garantizar a sus archivos las condiciones necesarias, en cuanto a edificios y equipamiento, de acuerdo a especificaciones técnicas». Para lograr esto establece además la profesionalización de los archivos al disponer que «a los efectos de garantizar la conservación y la organización de los documentos se propenderá a la profesionalización del personal responsable de los archivos». En relación con esto, se indagó sobre si el archivo está representado en el organigrama institucional, de quién depende administrativamente, qué funciones tiene asignadas por la institución, si 
tiene presupuesto asignado para cumplir esas funciones, cuántas personas trabajan en él y bajo qué modalidad contractual, cuál es el grado de instrucción máxima de ese personal asignado al archivo, qué escalafón y grado tienen los funcionarios, si en los próximos doce meses se prevé el ingreso de nuevo personal y, en caso que fuera afirmativo, con qué perfiles y bajo qué modalidad contractual sería incorporado.

3. Sobre el fondo documental. En este bloque de la encuesta se intentó conocer la situación actual de los archivos en cuanto al tratamiento técnico a grandes rasgos, con foco en el proceso de clasificación documental y en los cuadros de clasificación, y relevar si la institución posee un programa de gestión documental y, en caso de tenerlo, sobre la base de qué normativa se ha desarrollado; si existe un archivo central institucional y verificar que se haya realizado la identificación de series, el proceso de evaluación documental y elaboración de tablas de plazos precaucionales en consonancia con lo dispuesto por el artículo $6^{4}$ del Decreto 355/012 (2012) que regula la Ley de Sistema Nacional de Archivos (Ley 18220, 2007), y finalmente, y conocer si hay cuadro de clasificación en la institución. En caso de respuestas afirmativas sobre la presencia de un cuadro de clasificación, se indagó sobre el sistema de clasificación aplicado para su elaboración y sobre cuáles fueron la metodología y las fuentes de información utilizadas para elaborarlo. Asimismo, se buscó saber si era el primer cuadro de clasificación que poseía la institución o si había tenido otras versiones formales. A su vez, se indagó sobre el tipo de codificación utilizada para la construcción de ese cuadro, para lo que se solicitó al encuestado que brindara acceso al menos a una parte de su cuadro de clasificación para una mejor visualización de la codificación y estructuración del cuadro. En caso de que las respuestas sobre la existencia de un cuadro de clasificación fueran negativas, se indagó por qué no se ha elaborado esta herramienta básica para la gestión archivística, para intentar reconocer si esas causas estaban relacionadas con la situación del archivo relevada en la sección 2 de la encuesta.

4. Sobre el acceso a la información pública. En este último tramo de la encuesta se optó por sondear el conocimiento que tienen los profesionales

4 Decreto 355/012, artículo 6: «Responsabilidad de las Instituciones. Será responsabilidad institucional: a) Contar con profesionales Archivólogos en los archivos y en las unidades de administración documental de todas las instituciones públicas. b) Realizar una organización científica de los documentos en soporte tradicional o electrónica, mediante la organización de los archivos de Gestión, Central e Histórico. c) Promover la generación de espacios físicos, equipamiento y materiales adecuados para el funcionamiento de los archivos así como mantener óptimas condiciones medioambientales y de seguridad. d) Crear un sistema de seguridad y control a fin de evitar la sustracción y salida ilegal de documentos de los archivos que forman parte del Sistema Nacional de Archivos. e) Ninguna institución podrá eliminar documentos sin la previa consulta al Archivo General de la Nación. f) No eliminar documentos sin la previa consulta a la Comisión de Evaluación Documental de la Nación, que funcionará en la órbita del Archivo General de la Nación. g) Crear una comisión de evaluación documental institucional, cuyo cometido será evaluar la documentación existente en la institución para dar cumplimiento a la normativa vigente en materia de archivos. h) Designar a uno de los miembros de la Comisión de Evaluación Documental Institucional para actuar como nexo con el Archivo General de la Nación y la Comisión de Evaluación Documental de la Nación». 
de archivo sobre las normas nacionales de acceso a la información pública y cuál es su percepción respecto a la relación de estas normas con la gestión archivística.

Las preguntas de esta sección se refirieron expresamente a conocer el grado de conocimiento y manejo de las normas de acceso a la información pública, y a saber si en el archivo se resguardan documentos clasificados como reservados, confidenciales o secretos de acuerdo con lo establecido en los artículos 8 a $10^{5}$ de la Ley 18381 (2008).

Este apartado refleja lo que expresan Ramón Alberch-Fugueras y José Ramón Cruz Mundet:

Por principio, el derecho a la documentación, como el derecho a la información, es universal y no admite excepciones, y todas las leyes al respecto así lo manifiestan, si bien el derecho a conocer choca con otros derechos que han de ser protegidos de la intromisión es necesario tomar las debidas cautelas. En este sentido rodas ellas las adoptan para evitar que se ponga en peligro la intimidad y la defensa del Estado (2008, p. 103).

El cuestionario fue diseñado con mayoría de preguntas de múltiple opción, dejando siempre un espacio para observaciones que el encuestado entendiera necesarias hacer.

\section{Presentación de resultados}

La Ley 18220, promulgada el 20 de diciembre de 2007, que crea el Sistema Nacional de Archivos (SNA), establece en su artículo 4 el ámbito de su aplicación y define que «el alcance de este derecho obliga a todos los

5 «Artículo 8. (Excepciones a la información pública). Las excepciones a la información pública serán de interpretación estricta y comprenderán aquellas definidas como secretas por la ley y las que se definan seguidamente como de carácter reservado y confidencial.

Artículo 9. (Información reservada). Como información reservada podrá clasificarse aquella cuya difusión pueda: A) Comprometer la seguridad pública o la defensa nacional. B) Menoscabar la conducción de las negociaciones o bien, de las relaciones internacionales, incluida aquella información que otros estados $\mathrm{u}$ organismos internacionales entreguen con carácter de reservado al Estado uruguayo. C) Dañar la estabilidad financiera, económica o monetaria del país. D) Poner en riesgo la vida, la dignidad humana, la seguridad o la salud de cualquier persona. E) Suponer una pérdida de ventajas competitivas para el sujeto obligado o pueda dañar su proceso de producción. F) Desproteger descubrimientos científicos, tecnológicos o culturales desarrollados o en poder de los sujetos obligados. G) Afectar la provisión libre y franca de asesoramientos, opiniones o recomendaciones que formen parte del proceso deliberativo de los sujetos obligados hasta que sea adoptada la decisión respectiva, la cual deberá estar documentada.

Artículo 10. (Información confidencial). Se considera información confidencial: I) Aquella entregada en tal carácter a los sujetos obligados, siempre que: A) Refiera al patrimonio de la persona. B) Comprenda hechos o actos de carácter económico, contable, jurídico o administrativo, relativos a una persona física o jurídica, que pudiera ser útil para un competidor. C) Esté amparada por una cláusula contractual de confidencialidad.

II) Los datos personales que requieran previo consentimiento informado. Tendrán el mismo carácter los documentos o secciones de documentos que contengan estos datos.» 
órganos y organismos públicos, sean estatales o no estatales» (Ley 18220, 2007).

En el Decreto 355/012 del año 2012, que regula la ley mencionada, se describe específicamente a esos sujetos obligados en el literal a) del artículo 7, como los «pertenecientes a todos los poderes del Estado, instituciones públicas, entes autónomos, servicios descentralizados, gobiernos departamentales, municipios locales y personas públicas no estatales» (Decreto 355/012, 2012).

A partir de la encuesta aplicada, se pudo conocer el estado del tratamiento archivístico de los archivos pertenecientes al SNA, así como el estado de cumplimiento de la norma referida.

\subsection{Análisis FODA de la situación}

Una vez conocidos los resultados de la encuesta, es oportuno reflejarlos a través de una herramienta que permita visualizar de manera ordenada el estado de situación.

La herramienta seleccionada para ello es el Análisis FODA (o DAFO, por sus siglas en inglés), desarrollado por Albert Humpherey, que permite identificar y enumerar de manera sencilla y rápida las fortalezas, oportunidades, debilidades y amenazas de una determinada situación y, a partir de ellas, tomar decisiones.

En este caso particular el análisis e identificación de factores se da en virtud de los datos obtenidos mediante la aplicación de la encuesta a la muestra propuesta del total de sujetos obligados de la Ley 18220 (2007).

Aplicada esta herramienta, se identificaron los siguientes factores en la realidad de los archivos encuestados (ver figura 2): 


\begin{tabular}{|c|c|}
\hline FORTALEZAS & OPORTUNIDADES \\
\hline $\begin{array}{l}\text { Se visualiza una tendencia a profesionalizar } \\
\text { los archivos. }\end{array}$ & $\begin{array}{l}\text { La mayoría de los archivos relevados } \\
\text { dependen de una oficina directiva. }\end{array}$ \\
\hline $\begin{array}{l}\text { Existencia de normativa que regula el } \\
\text { Sistema Nacional de Archivos, y Acceso a } \\
\text { la Información Pública. }\end{array}$ & $\begin{array}{l}\text { Un } 8 \% \text { de los archivos está en proceso de } \\
\text { desarrollo de su Cuadro de Clasificación }\end{array}$ \\
\hline $\begin{array}{l}\text { Existe clasificación de la información de } \\
\text { acuerdo a lo establecido en la ley de acceso } \\
\text { a la información pública. }\end{array}$ & $\begin{array}{l}\text { Existencia de legislación referente al } \\
\text { Sistema Nacional de Archivos, y acceso a la } \\
\text { información pública. }\end{array}$ \\
\hline $\begin{array}{l}\text { Se posee acceso a varias herramientas y } \\
\text { fuentes institucionales para realizar una } \\
\text { identificación previa de la institución. }\end{array}$ & $\begin{array}{l}\text { La mayoría de los encuestados entienden } \\
\text { que hay una relación estrecha entre la } \\
\text { organización documental, la transparencia, } \\
\text { la rendición de cuentas y el acceso a la } \\
\text { información pública. }\end{array}$ \\
\hline $\begin{array}{l}\text { Hay un alto conocimiento de la normativa } \\
\text { nacional referente al sistema nacional de } \\
\text { archivos y al acceso a la información } \\
\text { pública. }\end{array}$ & $\begin{array}{l}\text { Se percibe entre los encuestados la } \\
\text { inquietud y necesidad de contar con } \\
\text { archivos organizados profesionalmente para } \\
\text { garantizar el acceso a la información } \\
\text { pública. }\end{array}$ \\
\hline & $\begin{array}{l}\text { Hay una clara percepción de la necesidad de } \\
\text { contar con Cuadros de clasificación como } \\
\text { herramienta, entre otras cosas, que facilita el } \\
\text { acceso a la información. }\end{array}$ \\
\hline DEBILIDADES & AMENAZAS \\
\hline $\begin{array}{l}\text { Sólo el } 38 \% \text { de los archivos posee Cuadro } \\
\text { de Clasificación. }\end{array}$ & $\begin{array}{l}\text { Al menos uno de los encuestados considera } \\
\text { que el Cuadro de Clasificación es } \\
\text { innecesario. }\end{array}$ \\
\hline $\begin{array}{l}\text { No existe una metodología estandarizada a } \\
\text { nivel nacional para la elaboración de los } \\
\text { Cuadros de clasificación. }\end{array}$ & $\begin{array}{l}\text { Falta de recursos, falta de personal, falta de } \\
\text { tiempo para atender a la elaboración de un } \\
\text { Cuadro de Clasificación. }\end{array}$ \\
\hline $\begin{array}{l}\text { El } 56 \% \text { de los casos no posee Cuadro de } \\
\text { Clasificación. }\end{array}$ & $\begin{array}{l}\text { Existencia de jefaturas no técnicas, lo que } \\
\text { en algunos casos impide una correcta } \\
\text { gestión archivística. }\end{array}$ \\
\hline $\begin{array}{l}\text { Escasez de personal. Hay un promedio de } 4 \\
\text { trabajadores por archivo en instituciones de } \\
\text { gran porte como Ministerios. }\end{array}$ & $\begin{array}{l}\text { En alguna situación se encontró que el } \\
\text { archivo ha sido eliminado del organigrama. }\end{array}$ \\
\hline $\begin{array}{l}\text { Solamente uno de los archivos cuenta con } \\
\text { tabla de plazos precaucionales aprobada por } \\
\text { la Comisión de Evaluación Documental de } \\
\text { la Nación. }\end{array}$ & $\begin{array}{l}\text { En algunas situaciones los profesionales que } \\
\text { ejercen en estos archivos no tienen } \\
\text { reconocido por sus autoridades } \\
\text { institucionales su titulación, ocupando } \\
\text { cargos administrativos o de servicio. }\end{array}$ \\
\hline \multicolumn{2}{|l|}{$\begin{array}{l}\text { En el } 55 \% \text { de los casos el archivo no se } \\
\text { visualiza en el organigrama. }\end{array}$} \\
\hline \multicolumn{2}{|l|}{$\begin{array}{l}\text { Falta de profesionales archivólogos al frente } \\
\text { de los archivos institucionales. }\end{array}$} \\
\hline $\begin{array}{l}\text { Escasa confianza en los sistemas de } \\
\text { clasificación funcionales. Sólo un } 11 \% \text { de } \\
\text { los archivos poseen un cuadro funcional. Un } \\
78 \% \text { utiliza cuadros orgánico-funcionales y } \\
\text { el } 11 \% \text { restante cuadros orgánicos. }\end{array}$ & \\
\hline
\end{tabular}

Figura 2.

Análisis FODA de la muestra relevada. Elaboración propia.

Como se puede observar, existen algunos factores que constituyen amenazas y debilidades sobre las cuales se debe trabajar para revertirlas. No obstante, 
hay una enumeración de factores reconocidos como fortalezas y oportunidades por perfeccionar. Ello demarca un terreno bastante favorable para seguir avanzando y para lograr objetivos que tiendan a alcanzar un afianzamiento y reconocimiento de la importancia de la gestión documental y archivística en las instituciones públicas de Uruguay, lo que permitirá que más archivos cuenten con profesionales y derivará en la incorporación de nuevas herramientas y procedimientos a las gestiones institucionales, entre ellas, los cuadros de clasificación de fondos documentales.

Ahora se dispone de datos que permiten visualizar y comprender el estado de situación de la clasificación documental en Uruguay, acotados a los archivos pertenecientes a los sujetos obligados de la Ley 18220 (2007).

Un $37 \%$ de los archivos pertenecientes a la muestra posee cuadro de clasificación, mientras que un $7 \%$ está en proceso de desarrollar uno y el restante $56 \%$ no lo tiene. Para este último grupo de archivos, entre los factores predominantes en la causa de la no existencia de un cuadro de clasificación se encuentran la falta de personal y de tiempo del personal existente, que debe cubrir varias tareas en su jornada laboral, por lo que carece de espacio en su rutina diaria para dedicarle tiempo de calidad a la planificación y elaboración de un cuadro de clasificación.

Si se llevan estos porcentajes al total de archivos pertenecientes al SNA, la cantidad de ellos que posee un cuadro de clasificación representa únicamente un 5,68\% del total de los sujetos obligados, mientras que un $1,13 \%$ del total está en proceso de elaboración, y un 8,33\% conoce la herramienta, pero al momento no la han desarrollado ${ }^{6}$.

La suma de estos porcentajes representa el 15,14\% del total de los sujetos obligados donde se cuenta con archivólogos, de los cuales, el $62 \%$ tiene reconocido su cargo como profesional, técnico o personal especializado, de acuerdo al escalafón de cada organismo.

Es clara la evidencia de que es necesario contar con más profesionales a cargo de la dirección técnica de los archivos, como disponen los artículos 1 y 7 de la Ley 18220 (2007). En la medida en que esto suceda, aumentará el número de archivos que tengan cuadros de clasificación y tablas de plazos precaucionales, entre otras cosas.

Sin duda, la situación descripta debe revertirse a la brevedad, en lo que será de gran importancia la promulgación de la Ley de Profesionalización de los Archivos, que se encuentra a estudio del Poder Legislativo ${ }^{7}$.

\footnotetext{
6 Esta realidad se supone a partir del conocimiento que se tiene de las instituciones, sus archivos y los profesionales archivólogos que trabajan en ellos. Debe recordarse que para el presente estudio se encuestaron a 40 profesionales que representan casi la totalidad de archivos que cuentan con archivólogos en el ámbito público de Uruguay. Es claro que sin archivólogo en una institución no hay gestión archivística del fondo documental, por lo cual, entre otras cosas, no hay cuadros de clasificación. De todas maneras, estas cifras quedan supeditadas a la posibilidad de realizar un estudio más profundo que abarque al total de los sujetos obligados por la Ley del Sistema Nacional de Archivos.

7 Disponible en : https://parlamento.gub.uy/documentosyleyes/ficha-asunto/139585
} 


\subsection{Propuesta de pautas para la clasificación y elaboración de cuadros de clasificación en el marco del Sistema Nacional de Archivos de Uruguay}

Ante las dificultades identificadas en la etapa de diagnóstico respecto de la situación actual de los archivos pertenecientes al SNA de Uruguay, como las debilidades y amenazas detectadas a través del análisis FODA aplicado (ver figura 2), es clara la necesidad de establecer pautas generales de fácil aplicación en los archivos públicos y en aquellos que no pertenecen al SNA pero quieran hacer uso de ellas.

Como ya es sabido, la clasificación funcional es la más estable y la más aceptada en la actualidad. En los últimos años se ha adoptado a nivel mundial este sistema de clasificación como el más aconsejable, y los teóricos llevan varios años intentando demostrar su conveniencia, basados esencialmente en el principio de procedencia de los documentos de archivo.

Según expone Javier Barbadillo Alonso (2010), los antecedentes de la clasificación funcional se remontan a 1941, con Edward Campell, archivero estadounidense, quien, según un estudio de Stuart Orr (2009), considera la clasificación funcional de aplicación menos costosa más coherente y comprensible, tanto para el personal del archivo como para sus usuarios, concepción que en su momento no tuvo los seguidores necesarios para que prosperara ante el modelo de clasificación orgánico.

Luego, en 1956, destacan los fundamentos teóricos de Schellenberg (2003), quien menciona la clasificación funcional e identifica dos tipos de actividades: las «sustantivas» que hacen a la razón de ser de la institución, y las «facilitativas» de corte más administrativo, repetitivas y transversales a la mayoría de las instituciones, que colaboran para que las tares sustantivas puedan ser llevadas a cabo.

De acuerdo con Barbadillo Alonso (2010), esta concepción coincide con las bases lógicas de la clasificación propuesta por Roberge años más tarde, en 1985.

Por su parte, en España, de la mano de Antonia Heredia Herrera en su Manual de organización de fondos de corporaciones locales: el Archivo de la Diputación Provincial de Sevilla (1980), surge una propuesta de aplicación de un cuadro de clasificación funcional que toma como base los preceptos teóricos establecidos por Schellenberg (1956). En 1994, Pedro López Gómez incursiona nuevamente en una propuesta de cuadro de clasificación funcional para los archivos históricos provinciales, buscando salvar problemas generados por los constantes cambios de estructuras orgánicas sobre las cuales se clasificaban los fondos documentales.

A principios del siglo XXI, las autoras Luciana Duranti y Elizabeth Shepherd determinan la clasificación funcional como la más acertada, como se ve en sus propias definiciones de clasificación y de cuadro de clasificación: «La 
clasificación es un medio de organizar e identificar todo los documentos entrantes, salientes e internos en relación con las acciones en las que participaron» (Duranti, 2002, p. 43). Con respecto a los esquemas de clasificación, entienden que «se basan en un análisis de funciones, procesos y actividades» (Shepherd, 2002, p. 73). Como menciona Barbadillo Alonso:

Las razones para preferir una clasificación funcional y no orgánica de las series parecen evidentes. Todo sistema de clasificación necesita un cierto grado de estabilidad. Las funciones que lleva a cabo una organización, aunque no sean estables por completo, lo son en mayor grado que su estructura organizativa, que cambia con frecuencia (2010, p. 97).

A su vez, José Ramón Cruz Mundet afirma:

Toda organización o entidad tiene unos fines u objetivos que le dan origen [...], en el desempeño de sus funciones, produce, entre otras, una actividad administrativa de la que se derivan los documentos, cuya organización debe reflejar ese proceso en el cual han sido creados (2015, p. 212).

Esto lo representa gráficamente en la siguiente figura:

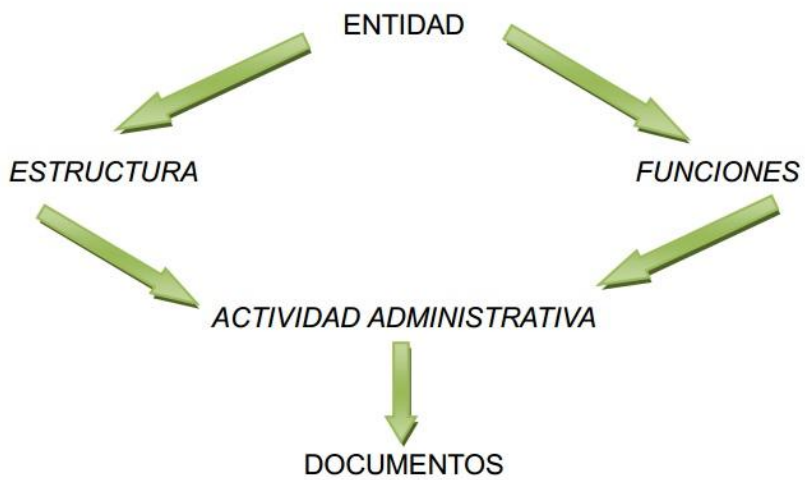

Figura 3.

Proceso de creación de documentos. Fuente: Cruz Mundet (2011, p. 213)

Estos documentos de archivo generados en función de una actividad administrativa pertenecen a una agrupación documental mayor, que es el fondo. Además, como recoge Cruz Mundet de las palabras de Duchein:

El sitio de un documento está en el seno de un proceso funcional, del cual es un elemento, por mínimo que sea. En un comienzo, nunca se lo concibe como un elemento aislado. Siempre posee un carácter utilitario que solo se hace claro cuando ha conservado el puesto que le corresponde dentro del conjunto de los demás documentos que lo acompañan (2011, p. 213).

Aquí Cruz Mundet deja claro que la clasificación de los documentos se encuentra íntimamente ligada con su procedencia, al afirmar lo siguiente: 
El respeto a la procedencia es, en definitiva, un método que proporciona una base segura tanto para el trabajo de clasificación como para la descripción de los fondos; todo lo obrado al margen del mismo es arbitrario, carente de rigor, subjetivo y, por consiguiente, antiarchivístico (2011, p. 213).

La clasificación funcional «trata de un criterio natural, puesto que emana de la naturaleza propia de los documentos y de los procesos que evidencian» (Red de Transparencia y Acceso a la Información, 2014). Esta explicación coincide con el esquema de representación de relaciones de las funciones respecto a las instituciones y los documentos de archivo que se encuentran en la Norma Internacional de Descripción de Funciones (ISDF) (Consejo Internacional de Archivos, 2007).

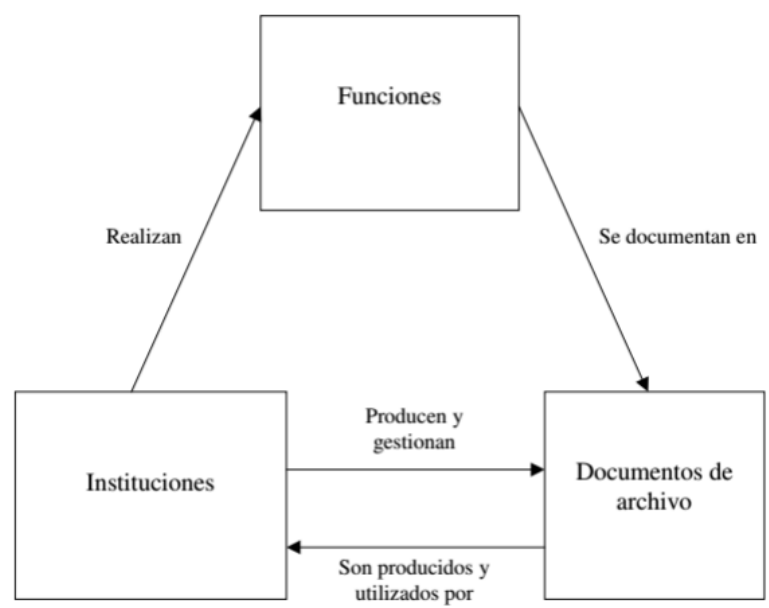

Figura 4.

Apéndice A. Representación del valor de las funciones con las instituciones y los documentos de archivo. Fuente: Norma ISDF (Consejo Internacional de Archivos, 2007, p.37)

Dicha representación no hace más que afirmar y graficar claramente la postura expuesta sobre la creación natural de los documentos de archivo en función del cumplimiento de los cometidos (funciones y actividades) de cualquier institución, por lo que es en los documentos que se evidencia la clasificación natural y funcional de fondos documentales. Esta noción es también reconocida como la más apropiada, e incluso se menciona como única por las normas ISO y otros documentos directivos, como el Modelo de Gestión Documental de la Red de Transparencia y Acceso a la Información (2014).

Ante la necesidad de contar con pautas que faciliten la tarea de clasificación y de elaboración de cuadros de clasificación funcionales a nivel nacional, siempre con el acceso a la información pública como meta, se ha estudiado la propuesta metodológica presentada por las archivistas españolas del Gobierno de Asturias Fernández Vega, Hernández Martín y De los Toyos de Castro (2014). Este trabajo, muy claro y práctico, establece una metodología para el desarrollo de cuadros de clasificación funcionales que toma como fuente primaria de información «los presupuestos generales de las organizaciones del sector público y los propios presupuestos generales de 
cada institución» (2014, p.195). Estos documentos normalizados resultan útiles en tanto establecen una

clasificación funcional del gasto adoptada en la elaboración de los presupuestos generales de las administraciones públicas que fija una estructura que recoge el conjunto de categorías y subcategorías funcionales atribuidas a cada organización. Esta estructura es la que servirá de soporte para identificar los niveles superiores del cuadro de clasificación (Fernández Vega, Hernández Martín y De los Toyos de Castro, 2014, p. 195).

Esto constituye un elemento útil y funcional para identificar las diferentes agrupaciones documentales que integrarán el cuadro de clasificación.

Si bien se trata de una metodología clara y fundamentada en criterios objetivos, en Uruguay los presupuestos no son elaborados de esta forma, sino por rubros generales a través de los cuales no se hace sencilla la identificación de las diferentes funciones y actividades de las instituciones, por lo que no es posible basar la clasificación documental en los presupuestos institucionales. Sin embargo, se cree conveniente tomar como base fuentes de información mencionadas, como las normas legales que establecen las funciones y actividades a cada institución y la normativa específica que regula los procedimientos administrativos, ya sean de carácter nacional o institucional.

En su propuesta, este grupo sugiere las diferentes categorías que han de componer el cuadro de clasificación, de acuerdo con la siguiente enumeración de reglas de procedimiento:

1. Identificación y denominación de la institución.

2. Identificación y denominación de la función.

3. Identificación y denominación de la subfunción.

4. Identificación de la actividad.

5. Identificación del procedimiento administrativo.

6. Identificación del tipo documental.

7. Identificación y denominación de la serie de documentos (Fernández Vega, Hernández Martín y De los Toyos de Castro, 2014, p. 197).

Estas reglas de procedimiento propuestas establecen

la sucesión de las acciones necesarias para identificar y formalizar las categorías lógicas y físicas que componen un sistema de clasificación funcional, mientras determinan la metodología para una correcta identificación, formalización y mantenimiento coherente del sistema a lo largo del tiempo, con la finalidad última de normalizar y documentar el proceso de clasificación en su conjunto (Fernández Vega, Hernández Martín y De los Toyos de Castro, 2014, p. 197).

Ante estos planteamientos es necesario recordar los principios de un cuadro de clasificación, como les llama Cruz Mundet (2011), a los que Heredia Herrera (2013) denomina «un conjunto de cualidades reconocibles»: 
- Delimitación: el cuadro de clasificación estará delimitado al conjunto orgánico de documentos producidos y recibidos por una institución en el ejercicio de sus funciones, esto es, al fondo. No se incluyen aquí, por ejemplo, colecciones que hayan ingresado al archivo por donación, compra, etc. Solo se admite un cuadro por fondo.

- Unicidad: este cuadro de clasificación abarcará, como se dijo, todo el fondo documental, con independencia de su cronología, esto es, desde el primer documento producido hasta los más recientes, hayan sido producidos en diferentes soportes o no.

- Estabilidad: debe garantizarse que ese cuadro de clasificación sea lo suficientemente estable para que perdure a lo largo del tiempo, recordando que el sistema de clasificación funcional es el que brinda mayor seguridad a la hora de cumplir con este principio. Esta estabilidad debe mantenerse al permitir la intercalación de nuevas series (flexibilidad).

- Simplificación: a efectos de asegurar la utilidad y éxito en la aplicación del cuadro de clasificación deben evitarse subdivisiones excesivas, procurando aplicar las divisiones justas y necesarias para clasificar el fondo. Esto facilita su elaboración, su comprensión y formalización.

De acuerdo con lo expuesto, se mencionan algunas pautas para la clasificación y elaboración de cuadros de clasificación para los archivos pertenecientes al SNA de Uruguay.

1. La clasificación de fondos se hará con base en las funciones y actividades del sujeto productor, respetando el principio de procedencia de los documentos.

2. Antes de llevar a cabo la clasificación es necesario realizar una identificación de la institución productora y de su fondo documental en cuatro niveles, a saber:

- Identificación y denominación de la institución productora de documentos (fondo). Aquí se deberá identificar a la institución productora del fondo documental, recordando que para que esta sea productora de un fondo documental tiene que cumplir con las cinco características enunciadas por Michel Duchein ${ }^{8}$ (1985, p. 76).

\footnotetext{
8 Esas características básicas son: tener un nombre y existencia jurídica, establecidos por documento legal que le da origen; tener atribuciones precisas y estables determinadas en el documento que le dio origen; ser parte de una estructura jerárquica con clara delimitación de subordinación; contar con un jefe/autoridad con poder de decisión; tener la estructura interna establecida formalmente a través de un organigrama.
} 
En esta etapa se obtiene conocimiento exhaustivo sobre la historia de la institución; sus funciones y actividades y su evolución a lo largo de la trayectoria institucional; la legislación y normativa (tanto nacional, internacional e interna) en la que se enmarca el ejercicio de las funciones institucionales; la estructura organizativa de la institución, su organigrama actual, así como los que ha tenido a lo largo de historia; la identificación de las diferentes autoridades que han ejercido la dirección de la institución.

Este conocimiento es necesario para tener claro que todo se verá reflejado en el fondo documental y que hará que su tratamiento sea más efectivo al estar enterados de antemano de cómo este se conforma. Esta información dará pautas para el establecimiento de diferentes procesos y políticas archivísticas por implementar a futuro.

- Identificación de las funciones de la institución productora. Aquí es importante identificar las grandes funciones o funciones mayores que se ejercen dentro de la institución productora. Estas estarán delimitadas a un grupo de actividades y procedimientos de diferentes ámbitos profesionales, que dependerán de la naturaleza de cada institución y estarán regulados por legislación específica.

Se encuentran funciones y procesos que serán transversales a todas las instituciones, como pueden ser la gestión de recursos humanos, la administración, las finanzas o la asesoría jurídica, entre otras, y otras más que serán exclusivas de cada institución de acuerdo a su ámbito de ejercicio.

- Identificación de las actividades de la institución productora. En este punto se pretenden identificar las actividades que se generan dentro de las funciones delimitadas en la etapa anterior. Son las actividades que permiten que la función pueda cumplirse. En algunos casos serán actividades de índole profesional y técnico (sustantivas) y en otro de índole administrativo y netamente de apoyo (facilitativas). Lo importante es poder identificar claramente la subordinación de estas con las determinadas en el nivel anterior.

- Identificación de las series documentales (serie). Aquí se pretende identificar todas las series documentales producidas por la institución, en el entendido de que las series son un «conjunto de documentos producido de manera continuada en el tiempo como resultado de una misma actividad y regulada por una norma de procedimiento», definición de José Ramón Cruz Mundet, tomada en el Glosario de las directrices de evaluación documental a nivel nacional publicadas por el Archivo General de la Nación de Uruguay en su primera edición de 2014. 
Para identificar las series se recomienda utilizar el formulario tipo elaborado por la Comisión de Evaluación Documental de la Nación, herramienta a disposición de los sujetos obligados en el sitio web del Archivo General de la Nación, y que también ha sido incluida tanto en la publicación de directrices antes mencionada, como en los temarios de talleres impartidos por esta comisión a los referentes a las diferentes instituciones parte del SNA, por lo que es un instrumento ya conocido y con buena aceptación por facilitar la tarea de identificación de series.

3. Se establecerán tres niveles en el cuadro de clasificación a partir del fondo: división del fondo que corresponde a la función, subdivisión del fondo concerniente a la actividad y serie. Podrá generarse también un cuarto nivel referido a la subserie. Estos niveles permitirán reflejar las relaciones existentes entre series, actividades y funciones.

La denominación establecida para cada nivel debe ser consistente, sencilla, representativa y lo más objetiva posible a efectos de evitar varias interpretaciones. Para ello se sugiere utilizar como títulos aquellos que deriven de los documentos normativos y que hayan servido de fuente de información para delimitar las diferentes agrupaciones.

4. En caso de encontrarse en el archivo con colecciones o archivos personales, estos no serán parte del cuadro de clasificación del fondo y, si su volumen lo amerita, cada uno de ellos tendrá su propio cuadro de clasificación

5. La codificación que se utilizará será alfanumérica, con el siguiente esquema que toma los niveles establecidos por la Norma Uruguaya de Descripción Archivística (nuda) (Archivo General de la Nación, 2016):
A100 Subfondo
A 200 Subfondo subordinado (si lo hubiera) ${ }^{9}$
A 400 Serie
B 100 Subfondo
B 200 Subfondo subordinado
B300 Subfondo subordinado subsidiario (si lo hubiera)
B 400 Serie

6. Una vez elaborado el cuadro de clasificación, este debe ser formalizado mediante su aprobación por parte de la autoridad máxima de la institución, a fin de poder establecer su aplicación en toda la institución, donde se aplicará desde la propia creación del documento.

\footnotetext{
«Cuando se nominen niveles intermedios a los definidos, deberá indicarse su relación o correspondencia con alguno de los niveles establecidos como básicos.» (Archivo General de la Nación, 2016, p. 27.) En este caso, dicha relación queda ilustrada en la codificación, ya que toma el primer digito del código del subfondo al que pertenece.
} 
En los documentos electrónicos y digitales, el código del cuadro de clasificación debe ser incorporado en sus metadatos.

7. Se desarrollarán tareas de asesoría y capacitación a todo el personal de la institución para que esté familiarizado con la aplicación de esta herramienta, que será una más dentro del flujo de procedimientos institucionales.

8. Es necesario que un equipo de expertos garantice la revisión periódica del cuadro para que sea actualizado en la medida en que puedan surgir nuevas series.

9. Si bien el cuadro de clasificación es una herramienta estrictamente archivística y su elaboración estará a cargo del profesional archivólogo de la institución, no debe descartarse que este se asesore con profesionales de otras áreas, como juristas o informáticos, para garantizar una comprensión y aplicación universal a nivel institucional.

\section{Consideraciones finales}

En la gestión de archivos, como sucede en todas las disciplinas, es necesario contar con legislación, sistemas y presupuesto que permitan llevar a cabo las tareas profesionales que en su conjunto tienen como finalidad el lograr una óptima gestión, administración, conservación y difusión de la información contenida en los archivos.

En este caso, se ha trabajado especialmente sobre la clasificación como parte primordial en esa gestión. Se vio que de la aplicación de la clasificación surge el cuadro de clasificación, que debe ser elaborado a partir de criterios funcionales, como ya se estableció con sobrados motivos. Podemos afirmar que este cuadro de clasificación «es el cuore no solo del proceso de clasificación sino de la gestión integral de los sistemas electrónicos de gestión de documentos» (Díaz Rodríguez, 2010), a lo cual podría agregarse que lo es de los sistemas de gestión documental en general, o, como expresa Heredia Herrera: «La clasificación siguiendo el principio de procedencia precisa de su plasmación material en un esquema o cuadro que no es otra cosa que el andamio para sistematizar cada fondo» (1991, p. 267).

Esta justificación sobre la importancia del cuadro de clasificación como herramienta de gestión es base y soporte para el desarrollo del resto de las tareas y técnicas que se aplicarán al fondo documental de interés aquí.

A través del presente estudio se identificó que en Uruguay los archivos cuentan con legislación y pertenecen a un sistema nacional. Ahora, faltaría obtener mayores presupuestos y garantizar su gestión profesional. Heredia Herrera lo expresa claramente:

Los dos pilares primeros en los que ha de basarse una administración de archivos: el sistema de archivos y la legislación, pero ya dijimos 
que ambos precisan del apoyo de unas dotaciones económicas que den efectividad a aquellos y no distancien la teoría de la práctica, y de la existencia de unos técnicos suficientes y cualificados que en gran parte también dependen del soporte presupuestario (1991, p. 216).

Esto es claro: sin presupuesto no hay profesionales y sin profesionales no hay gestión que garantice el cumplimiento de la legislación ni de las directrices de ningún SNA. Se pudo observar que, en los casos donde hay profesionales, si son pocos, les resulta muy difícil dedicar tiempo a la planificación y desarrollo de la clasificación porque deben dedicar sus horas y esfuerzos a atender otras solicitudes.

Las pautas propuestas aquí pretenden ser una modesta ayuda para facilitar la tarea de clasificación, ya que enumeran tareas concretas y detalles importantes que no se pueden obviar para aprovechar al máximo los recursos disponibles: «... al no existir unas orientaciones sobre equipamientos y sobre lo que es más importante criterios de organización y descripción, suelen gastarse mal. Esta ausencia de normas, de orientaciones, puede ocasionar errores irremediables» (Heredia Herrera, 1991, p. 217).

Autores como Alberch-Fugueras y Cruz Mundet han ratificado lo que se ha expresado aquí:

Los archiveros nos encontramos, pues, con el desafío [...] de hacer accesible la información contenida tanto en el documento papel como en el documento electrónico, [...] y con la imperiosa necesidad de recursos económicos para evitar que una parte relevante de la memoria colectiva se convierta en memoria efímera, hecho que equivaldría a condenar a los ciudadanos a perder sus raíces y su identidad (2008, p. 166).

Se sostiene la convicción que las autoridades y la ciudadanía en general tienen por concepción que el archivo es un depósito lleno de papeles viejos empolvados y entienden que poco rédito se puede sacar de ellos. Pues como es notorio, esa concepción es totalmente errónea. Una gestión profesional y eficiente de los archivos puede traer aparejada una serie de beneficios, tanto administrativos y de gestión, como económicos. Es allí a donde debe apuntarse para terminar de hacer entender de una vez por todas, el valor que resguardan los archivos, y no solo desde el punto de vista patrimonial. Es muchísimo más de lo imaginado lo que se consigue ahorrar teniendo una gestión archivística profesional con implantación de un Sistema de Gestión de Documentos, entre ellos destaca la rápida de recuperación de la información, evitando la excesiva pérdida de horas hombres para encontrar los documentos solicitados, tiempo que, entre otras cosas, logra dedicar a la planificación y aplicación de la clasificación documental. También se pueden lograr beneficios significativos en los costos insumidos por la pérdida de litigios contra la administración, por el hecho de no contar o no encontrar las pruebas documentales necesarias en tiempo y forma. Y sin duda el ahorro mayor es generado en el alquiler de locales que ofician de depósitos de documentos, o la tercerización de la custodia de los documentos, gastando la gran parte de las veces excesivas sumas de dinero en guardar documentación que 
ya no posee valor secundario, o que es fácilmente recuperable en diversas fuentes de información (Alberch-Fugueras, 2013, p. 60).

Si bien se ha visto que hay una gestión archivística que intenta ser lo más eficiente posible con los medios que posee, a diez años de la promulgación de la Ley del Sistema Nacional de Archivos, se entiende que las instituciones que poseen archivólogos e instrumentos de gestión archivista como el cuadro de clasificación, los archivos que lo han elaborado y los archivos que poseen profesionales en su dirección deberían ser muchos más.

En Uruguay la archivística se ha desarrollado significativamente, y esto obedece en gran parte a la existencia de legislación a nivel nacional, que es el caballito de batalla cotidiano de los archivistas en el ejercicio de su profesión, siendo de mucha ayuda para que prosperen proyectos que hoy son realidad.

Quizás a futuro este trabajo pueda suscitar un análisis mayor de la propuesta aquí presentada en la órbita del SNA, a efectos de definir formalmente directrices específicas para la clasificación documental a nivel nacional, recordando que la ley de archivos y su decreto reglamentario prevén que el «Archivo General de la Nación formará grupos de trabajo con profesionales Archivólogos en las diferentes especialidades» y que «será el órgano rector de la política archivística nacional tendiente a: normalización, diseño y ejecución de las políticas y asesorar en la gestión documental y archivística» (artículo 4 del Decreto 355/012, 2012). Es claro que esto recién comienza y que se debe seguir trabajando en pos de la salvaguarda del patrimonio documental de Uruguay y del mundo, teniendo siempre presente que el usuario final es el ciudadano, el mismo que cada vez toma más fuerza para exigir la democracia en su país, así como una transparencia en el accionar de las instituciones públicas que son gestionadas con el aporte de sus impuestos.

Ello lleva a exigir del Estado una rendición de cuentas constante y, algo no menor, la participación ciudadana, pilares sobre los que funciona el gobierno abierto, del cual tanto se habla en Latinoamérica. Lo cierto es que sin archivos gestionados profesionalmente no hay acceso a la información y sin él no hay rendición de cuentas ni transparencia, por lo cual se concluye que el gobierno abierto y el Estado deben comenzar a mirar con otros ojos la gestión documental, dándole la importancia que posee y otorgando los recursos necesarios que garanticen su gestión de acuerdo a la normativa nacional.

La ciencia archivística es una aliada de la democracia al construir una garantía del pleno ejercicio de los derechos ciudadanos (acceso a la información, toma de conciencia de la identidad y el sentido de pertenencia a las colectividades), es un factor de eficiencia, eficacia y rentabilidad de las organizaciones públicas y privadas, un sector comprometido en el uso dinámico y sensato de las tecnologías de la información y la comunicación (Alberch-Fugueras, 2013, p. 91). 


\section{Referencias bibliográficas}

ALBERCH-FUGUERAS, R. (2013). Archivos. Barcelona: UOC.

ALBERCH-FUGUERAS, R.; CRUZ-MUNDET, J. R. (2008). ;Archívese! Los documentos del poder. El poder de los documentos. 3. a edición. Madrid: Alianza.

ARCHIVO GENERAL DE LA NACIÓN (2016). Norma Uruguaya de Descripción Archivística. Montevideo: Archivo General de la Nación.

BARBADILLO ALONSO, J. (2010). Clasificaciones y relaciones funcionales de los documentos de archivo. Tabula, (13), 95-112. Recuperado de http://revistasacal.datalib.es/index.php/tabula/article/view/244

\section{COMISIÓN DE EVALUACIÓN DOCUMENTAL DE LA NACIÓN} (2016). Directrices generales para la evaluación documental a nivel nacional. 2. ${ }^{\text {a }}$ edición. Montevideo: Archivo General de la Nación.

CONSEJO INTERNACIONAL DE ARCHIVOS. (2007). ISDF: Norma Internacional para la Descripción de Funciones. Elaborada por el Comité de Buenas Prácticas y Normas Profesionales. Dresde, Alemania, 2-4 mayo 2007. Recuperado de http://www.mcu.es/ccbae/es/consulta/registro.cmd?id=213674\%5Cnht tp://www.ica.org/sites/default/files/CBPS_2007_Guidelines_ISDF_Fir st-edition_SP.pdf

CRUZ-MUNDET, J. R. (2011). Administración de documentos y archivos: textos fundamentales. Madrid: C. de A. de Archiveros. Recuperado de http://www.archiveros.net/LIBRO.ARCHIVOS.IBEROAMERICAN OS.pdf

CRUZ-MUNDET, J. R. (2015). Archivística. Gestión de documentos y administración de archivos. 2. ${ }^{a}$ edición. Madrid: Alianza.

DECRETO 70/015 (2015). Modificación del Art. 6 del Decreto 355/012 Relativo al Sistema Nacional de Archivos. Montevideo: Poder Legislativo. Recuperado de https://www.impo.com.uy/bases/decretos/70-2015

DECRETO 355/012 (2012). Reglamentación de la Regulación de la Actividad Archivística, Creando el Sistema Nacional de Archivos. Montevideo: Poder Legislativo. Recuperado de https://www.impo.com.uy/bases/decretos/355-2012/14

DÍAZ RODRÍGUEZ, A. (2010). La clasificación como proceso de gestión de documentos. Tabula, (13), 79-93. 
FERNÁNDEZ VEGA, M. C.; HERNÁNDEZ MARTÍN, A.; DE LOS TOYOS DE CASTRO, A. B. (2014, noviembre). Normalizando la clasificación de documentos: propuesta de reglas. En Fundación Sierra Pambley (Ed.), Jornadas Archivando 7: La nueva Gestión de Archivos (pp. 193-207). León. Recuperado de https://archivofsierrapambley.files.wordpress.com/2015/02/gobierno_ asturias_2014.pdf

FOSCARINI, F. (2010). La clasificación de documentos basada en funciones: comparación de la teoría y la práctica. Tabula, (13), 41-57.

HEREDIA HERRERA, A. (1980). Manual de organización de fondos de corporaciones locales: el Archivo de la Diputación Provincial de Sevilla. Sevilla: Diputación Provincial. Archivo Organización España Subdirección General de Archivos.

HEREDIA HERRERA, A. (1986). Archivística general: teoría y práctica. Sevilla: D. P. de Sevilla.

HEREDIA HERRERA, A. (2011a). Lenguaje y vocabulario archivísticos. Algo más que un diccionario. Andalucía: Tecnographic- J. de A. C. de Cultura.

HEREDIA HERRERA, A. (2011b). La archivística, a debate. Boletín de la $A N A B A D, 3,7-26$. Recuperado de https://revistas.unc.edu.ar/index.php/anuario/article/view/12484

HEREDIA HERRERA, A. (2013). Manual de archivística básica: gestión y sistemas. Puebla: Benemérita Universidad Autónoma de Puebla - E. E. Editor.

LEY 18220 (2007). Sistema Nacional de Archivos. Conservación y Organización del Patrimonio Documental de la Nación. Montevideo: Poder Legislativo. Recuperado de https://www.impo.com.uy/bases/leyes/18220-2007

LEY 18381 (2008). Derecho de Acceso a la Información Pública. Montevideo: Poder Legislativo. Recuperado de https://www.impo.com.uy/bases/leyes/18381-2008/9

\section{ORGANIZACIÓN DE LAS NACIONES UNIDAS PARA LA} EDUCACIÓN (1985). La administración moderna de archivos y la gestión de documentos: el prontuario RAMP. París: Unesco.

\section{RED DE TRANSPARENCIA Y ACCESO A LA INFORMACIÓN (2014).}

Directrices. Identificación y clasificación. Modelo de Gestión de Documentos y Administración de Archivos para la Red de Transparencia y Acceso a la Información. Recuperado de http://mgd.redrta.org/directrices-identificacion-yclasificacion/mgd/2015-01-28/101617.html\#vtxt_cuerpo_T0 
SCHELLENBERG, T. R. (2003). Modern Archives: Principles and

Techniques. 3. ${ }^{\text {a }}$ edición. Chicago: S. of A. Archivists. Recuperado de http://www.archivists.org/publications\%0A/epubs/ModernArchivesSchellenberg.pdf

La corrección de estilo del presente artículo estuvo a cargo de Nairí Aharonián. 